5) Geffcken, G.: Z. Physik. Chem., 49, 257 (1904)

6) Gordon, V.: ibid., 18, 1 (1895)

7) Harned, H.S. and R. S. Davis Jr.: J. Am. Chem. Soc., 65 $2030(1943)$

8) International Critical Tables, McGraw-Hill, New York, 3, 271 (1928)

9) Kortü, G.: Z. Electrochem, 42, 287 (1936)

10) Landolt-Börnstein Tables, Springer, Berlin, II. Band 2, Teil, 1-1 (1962)

11) Long, F. A. and W. F. McDevit: Chem. Rev., 51, 119 (1952)

12) Markham, A.E. and K. A. Kobe: J. Am. Chem. Soc., 63,
$449(1941)$

13) Markham, A.E. and K. A. Kobe: ibid, 63, 1165 (1941)

14) Michels, A., J. Gerver and A. Bi1: Physica, 3, 797 (1936)

15) Morrison, T. J. and F. Billett: J. Chem. Soc., 3819 (1952)

16) Rothmund, V. : Löslichkeit und Löslichekeitsbeeinflussung.

Barth, Leipzig (1907)

17) Setschenow, J.: Z. Physik. Chem., 4, 117 (1889)

18) Tamman, G. : ibid, 11, 676 (1893)

19) van Krevelen, D. W. and P. J. Hoftijzer: Chim. Ind.

$\mathrm{XXI}^{\text {eme }}$ Congr. Int. Chim. Ind., 168 (1948)

20) Winkler, L. W.: Ber., 22, 1764 (1889)

\title{
LIQUID BEHAVIOUR IN BUBBLE COLUMN
}

\author{
EIICHI KUNUGITA, ${ }^{* *}$ MICHIO IKURA AND TSUTAO OTAKE \\ Department of Chemical Engineering, Faculty of \\ Engineering Science, Osaka Unitersity, Toyonaka, Osaka
}

\begin{abstract}
The motion of a solid particle which was allowed to drift in a liquid phase was pursued by a camera to find the following :

(1) There is no remarkable irregularity of liquid flow in the bubble column.

(2) The distribution function of axial displacements of a solid particle was discribed by the normal distribution function. The assumed dispersion model coincided well with the experimental results. From this fact the mechanism of the mixing of liquid element was considered to be analogous to that of diffusion. The dispersion coefficient was also determined experimentally.

(3) The dynamic behaviour of bubbles, especially the coalescence of bubbles, has the controlling effect on the gas holdup and the dispersion coefficient.
\end{abstract}

\section{Introduction}

Bubble columns are often used as gas-liquid reactors in many chemical industries. However, their chracteristics are not yet fully made clear. When the bubble column is used as a reactor, many problems such as the heat transfer between liquid and column wall, the mass transfer between liquid and bubbles, and the mixing of liquid and bubbles, become important.

Up to this time, every investigation of liquid mixing in the bubble column analyzed the axial dispersion model using sodium chloride or potassium chloride as a tracer. Siemes and Weiss ${ }^{8)}$ recorded the transient response to a liquid tracer pulse in a column without liquid phase flow. Tadaki and Maeda $^{9}$ analyzed liquid mixing in a column with countercurrent flow by the pulse response to a liquid tracer. Argo and Cova ${ }^{2)}$ carried out an investigation by measurement of the steady state concentration gradient of the tracer for continuous tracer injection with a continuous liquid flow. Bischoff and Phillips ${ }^{3)}$ used the same experimental method as that of Tadaki and Maeda ${ }^{9}$ in a concurrent column. Reith, Renken and Israel $^{7)}$, and Aoyama, Ogushi, Koide and Kubota ${ }^{17}$ measured the steady state concentration gradient of the tracer as in Argo and Cova's method ${ }^{22}$. Aoyama et al. "', moreover, used the heat which was generated by a nicrome wire as a tracer.

\footnotetext{
* Received on Apr. 30, 1969

** Dept. of Chem. Eng., Kobe Univ., Kobe
}

Except for the research of Aoyama et al. ${ }^{1)}$, all investigations were carried out using electrolyte as a tracer. Generally the bubble behaviour which causes liquid mixing in the column depends upon the conditions of the gas-liquid interface. For example, Koide, Kato, Tanaka and Kubota ${ }^{6)}$, and Braulick, Fair and Lerner ${ }^{4}$ recognized that the bubble size tends to become smaller by the addition of small amounts of electrolyte to the liquid phase as a tracer. In the present research, a solid particle was pursued by the camera. According to the particle motion observed, the degree of liquid mixing was analyzed using a statistical method.

\section{Experimental}

The experimental apparatus used is shown in Fig. 1 The column (Diameter; $50 \mathrm{~mm}$, Height $; 1000 \mathrm{~mm}$ ), made of clear acrylic plastics, was equipped with a porous plate as a gas distributor. This porous plate had the same diameter as that of the column and the pore size was $60 \mu$. To prevent distortion, the bubble column was surrounded by a clear acrylic plastic box of square section and the space between the box and the column was filled with water which was circulated from the thermostat.

Six mirrors were placed around the bubble column and adjusted to take two mutually perpendicular views of column simultaneously.

The flow patten could be detected by the motion of a solid particle. These optical systems are shown in 
Fig. 2. For the purpose of preventing the doubled views arising from the assorted optical systems, the surface of reflecting mirrors was coated with vacuum evaporated aluminum.

All experiments were carried out under the batch operation of the liquid phase. Ion exchanged water and air were used respectively as liquid and gas. The air was supplied to the column after purification by being passed through oil filters, concentrated sulfuric acid and caustic soda in turn.

A solid particle (Diameter; about $5 \mathrm{~mm}$, Specific gravity; 0.99 that was adjusted to that of water by inlaying a piece of wire to a rubber ball.) was set adrift. The view of it was pursued continuously by a camera which can roll up the film at short time intervals.

The aggregation or the coalescence of bubbles was also studied according to the film. The superficial gas velocity covered was from 0.76 to $8.0 \mathrm{~cm} / \mathrm{sec}$. The experimental temperature was kept at $20 \pm 1^{\circ} \mathrm{C}$.

The camera used was a Nikon F Motor Drive with telephoto lens Nikkor-Q Auto 1; 3.5 of $135 \mathrm{~mm}$ focal length. The film used was Fuji Neopan SS (ASA 100).

\section{Results}

\section{(1) Gas holdup}

The difference between aerated liquid level $L$ and clear one $L_{0}$ was measured and the gas holdup $\phi$ was calculated according to $\phi=\frac{L-L_{0}}{L} \times 100(\%)$. In Fig. 3 the gas holdup is plotted as a function of the superficial gas velocity with the results of other investigators ${ }^{1,2,5,7,9)}$.

\section{(2) Motion of a solid particle}

If there are no slips between a solid particle and the liquid, the motion of a solid particle will exactly represent that of a liquid element. Therefore, the average

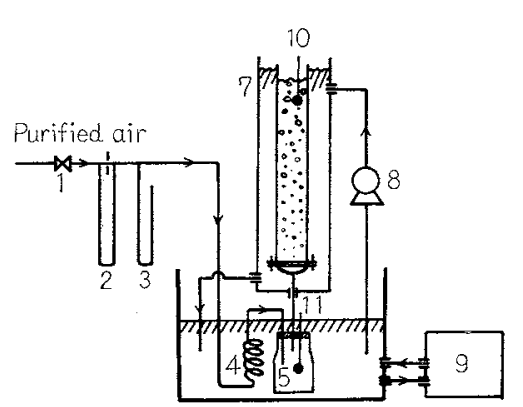

1) Control value

2) Orifice flow meter

3) Manometer

4) Gas preheater

5) Trap

6) Bubble column

7) Clear acrylic plastic box of square section

8) Circulation pump

9) Thermostat

10) Thermometer

11) Thermometer.

Fig. I Experimental apparatus

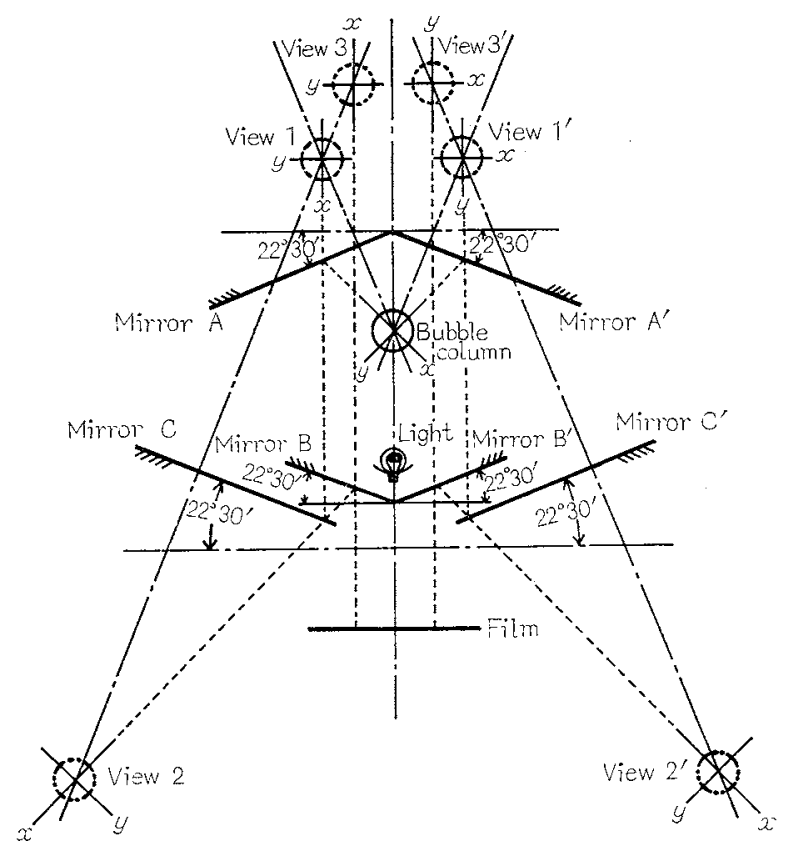

Fig. 2 Relationships of optical system and views
Fig. 3 Gas holdup as a function of the superficial gas velocity

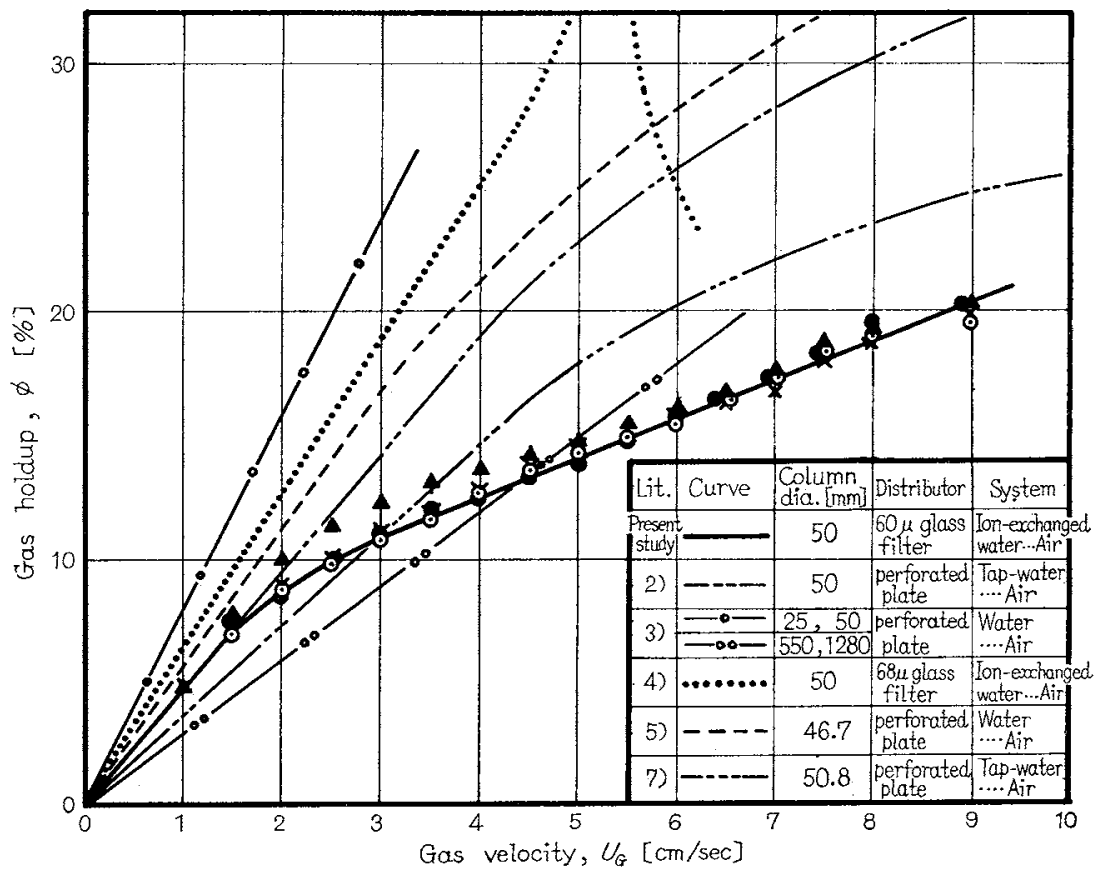


velocity of a solid particle at a certain position would be interpreted as the flow of the liquid phase. The axial velocity was computed from the difference of the distance in the picture for the every fixed short time interval. The radial position of the particle was determined using the relationship $r=\sqrt{x^{2}+y^{2}}$, where $x$ and $y$ are the mutually perpendicular distances from the center of the column. In this study, only the axial component of the velocity of a solid particle was considered for simplicity. If the distribution of the axial velocity in the radial direction can be detected, it will be made clear whether or not there is a deviation of liquid flow in the bubble column.

In Figs. 4 and 5, the axial velocity of a solid particle and their average value are shown. As shown in these figures, the whole cross section may be regarded to be in uniform turbulence, though there is a small down flow near the wall for low superficial gas velocity.

The motion of a solid particle in the radial direction was neglected and only the axial movement was considered. In Fig. 6 an example of axial motion is shown.

\section{Analysis}

The behaviour of a solid particle was divided into axial displacements within each fixed short time interval. This was fixed at one-third second, which was the rollup time of the film. About 100 to 150 periods of axial displacement were measured. Measurement of each displacement was made as follows. As is shown in Fig. 7,

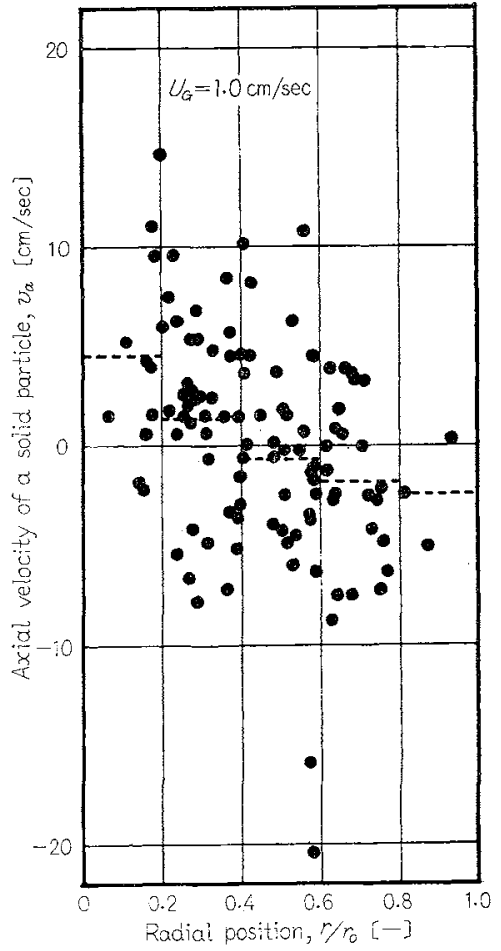

Fig. 4 Distribution of the axial velocity

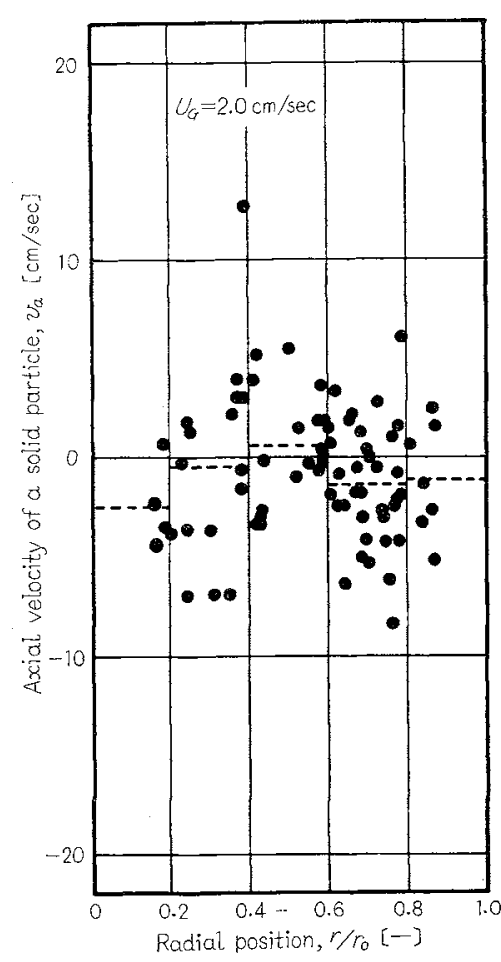

Fig. 5 Distribution of the axial velocity

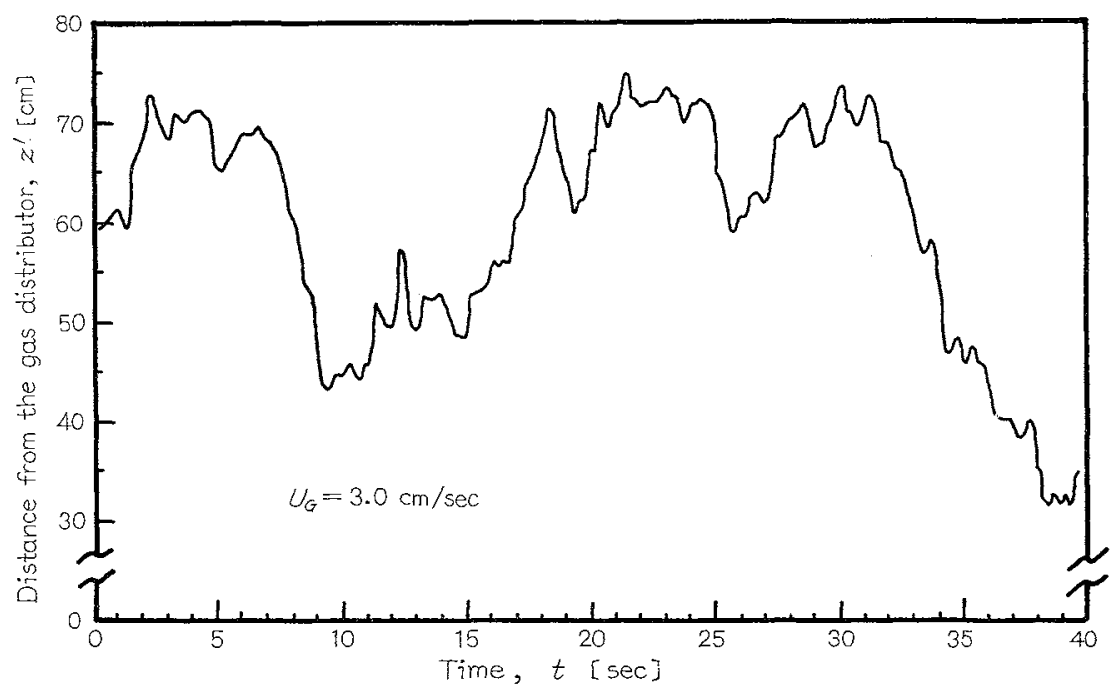

Fig. 6 Axial motion of a solid particle as a function of time 
the difference between initial position, $z_{i}^{\prime}$ at $t_{i}$, and final one, $z^{\prime}{ }_{i+1}$ at $t_{i+1}$, was regarded as the displacement within the $i$ th time interval, $\left(t_{i+1}-t_{i}\right)$. Then the final position of the $i$ th time interval was considered as the initial position of the next time interval, $\left(t_{i+2}-t_{i+1}\right)$. The same procedures were repeated for the following time interval.

The mesurement of 100 to 150 axial displacements of a solid particle corresponds to an experiment of throwing 100 to 150 particles into a cross section, $z=0$ in a moment and measuring how many of these particles may be found at a given axial position. Axial motion in Fig. 6 is converted into a histogram of displacement within the fixed short time interval as is shown in Fig. 8 . " $\tau$ " is the time interval, $\left(t_{i+1}-t_{i}\right)=\left(t_{i+2}-t_{i+1}\right)=\cdots \cdots$. At $\tau=0$ all of the particles should be concentrated at $z=0$.

The cumulation of this histogram is plotted on normal distribution paper, Fig. 9. The plot may be regarded as a straight line. It was found that almost every result was approximately linear on normal probability paper. That is, the distribution of axial displacements of a solid particle follows a normal probability distribution. This fact suggests that the mechanism of the mixing of liquid element is analogous to that of diffusion.

The breadth of the distribution of solid particle displacement represents the intensity of axial mixing of the liquid phase. This probability density $F$ is related to the concentration ratio as the follows:

$$
F=\frac{n}{\int n d z}=\frac{C}{C_{0} L} \quad[1 / \mathrm{cm}]
$$

$n$; number of solid particles in a unit volume of an apparatus. $\int n d z$; number of solid particles throughout an apparatus.

$z$; distance in an apparatus.

$L$; full length of an apparatus.

$C$; concentration of a liquid tracer at any position.

$C_{0}$; average concentration of a liquid tracer spreading throughout an apparatus.

According to the above consideration, the following differential equation is adaptable for liquid behaviour in a batch operation column.

$$
\begin{aligned}
& \partial F \\
& \partial \tau
\end{aligned}=E \frac{\partial^{2} F}{\partial z^{2}}
$$

If the bubble column were regarded as an infinite one, the boundary conditions should become as follows.

$$
\left.\begin{array}{rr}
\text { (1) } \quad F(z, 0)=0 & (-\infty<z<+\infty) \\
\text { (2) }-2 E^{\frac{\partial}{\partial z}}=\delta(\tau) & (z=0) \\
\text { (3) } \lim _{z \rightarrow-\infty} F(z, \tau)=0 & \lim _{z \rightarrow+\infty} F(z, \tau)=0 \\
& (\tau \geqq 0)
\end{array}\right\}
$$

The solution of Eq. (1) under the boundary condition (2) is

$$
F(z, \tau)=\frac{1}{2 \sqrt{\pi E \tau}} \exp \left(-\frac{z^{2}}{4 E_{\tau}}\right)
$$

Eq. (3) shows the distribution which is formed after $\tau$ second. In the present study $\tau$ corresponds to the

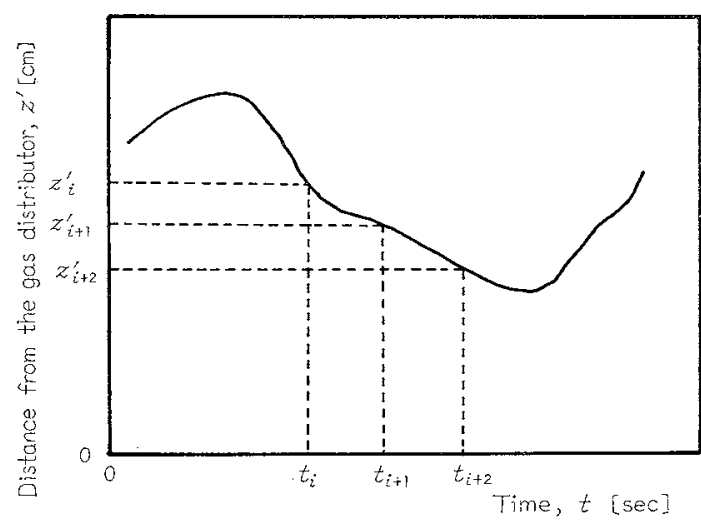

Fig. 7 Relationsphip between the axial displacement and the time interval

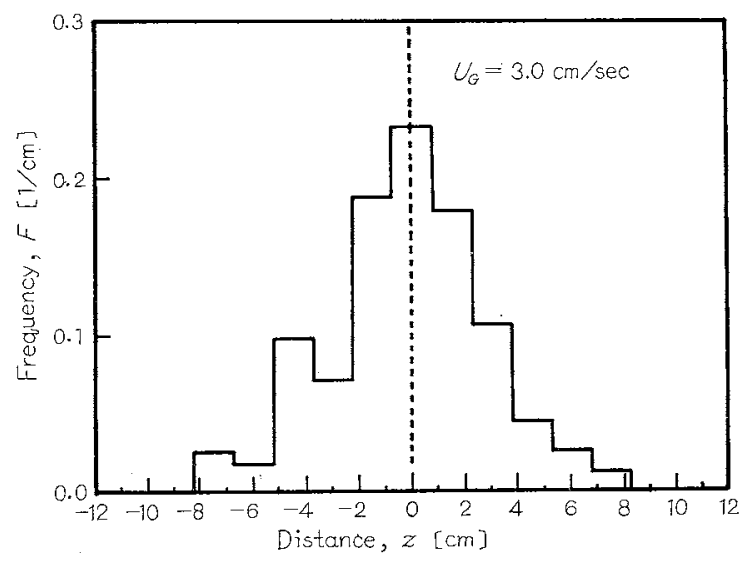

Fig. 8 Frequency distribution of displacements of a solid particle

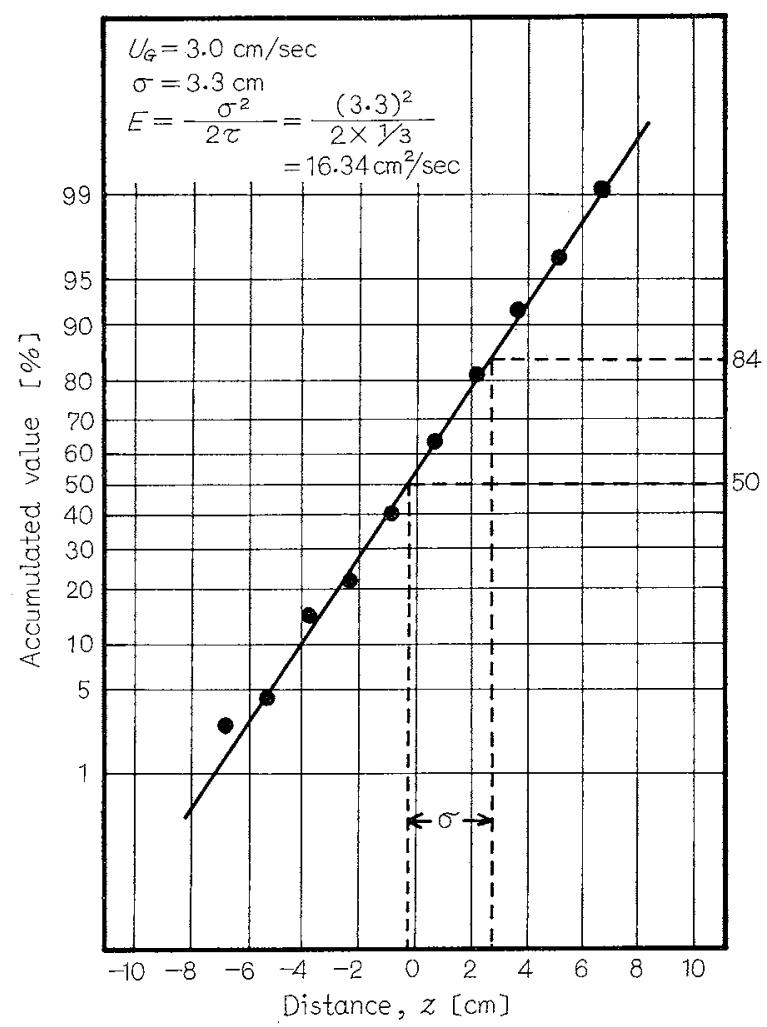

Fig. 9 Cumulation of a frequency distribution of displacements of a solid particle 
roll-up time of the film. Eq. (3) will be rearranged to the normal distribution function by substituting $\sigma^{2} / 2 \tau$ into $E$. The variance, $\sigma^{2}$ obtained from the experiment is a measure of the mixing and corresponds to $2 E \tau$. The coincidence between Eq. (3) and experimental results supports the concept that the mixing in the bubble column may be regarded to follow the axial dispersion model, Eq. (1). From the slope of the line on normal probability paper, " $\sigma$ " was estimated and then $E$ was calculated.

\section{Discussion}

\section{(1) Gas holdup}

It is reported that the gas holdup greatly depends upon the column diameter when that is smaller than $550 \mathrm{~mm}^{5}$. In Fig. 3 previous results are shown along with the result of the present study. Generally, when there are not so many interactions between bubbles, the gas holdup increases linearly with the superficial gas velocity.

The results of Tadaki and Maeda ${ }^{97}$ agrees well with that of the present study at low superficial gas velocity in spite of using a different type gas distributor. But it was found that the increasing rate of gas holdup in the present study decreases at about $2 \mathrm{~cm} / \mathrm{sec}$ of the superficial gas velocity. It was observed from the photos that at about $2 \mathrm{~cm} / \mathrm{sec}$ aggregation or coalescence of bubbles occured. According to the plots, in the case of Tadaki and Maeda $^{9)}$ this critical superficial gas velocity was found to be $4 \mathrm{~cm} / \mathrm{sec}$.

On the contrary, the result of Aoyama et $\mathrm{al}^{\mathrm{i}}{ }^{\text {s }}$ shows a larger gas holdup than that of the present study in

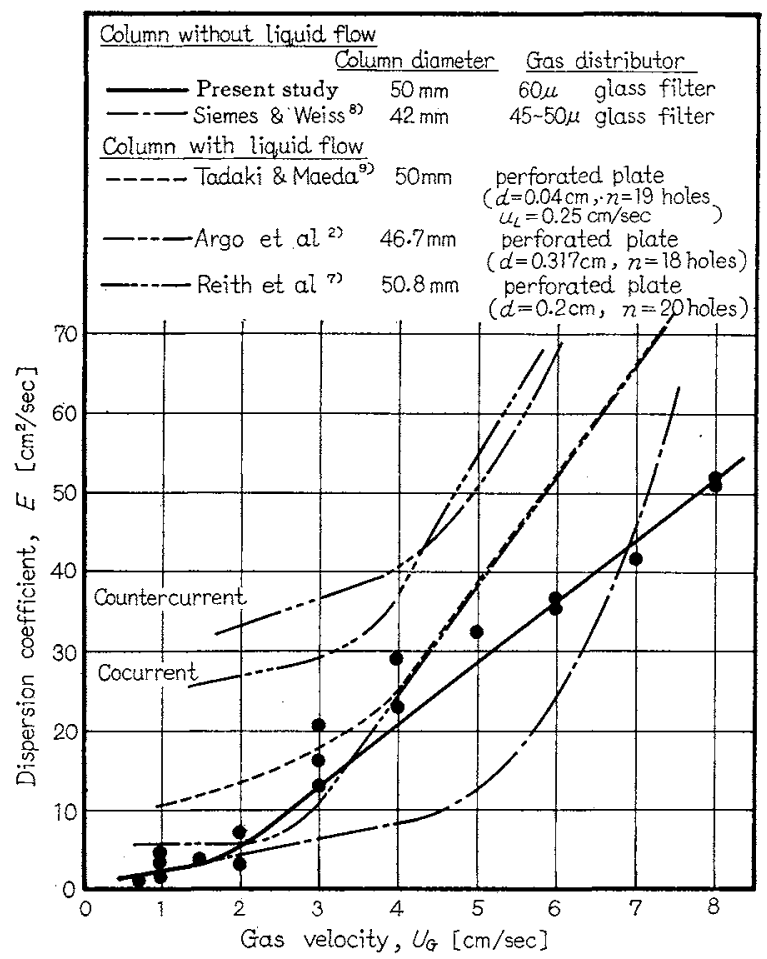

Fig. 10 Axial dispersion coefficient as a function of the superficial gas velocity spite of using almost the same gas distributor and the same column diameter. Their critical superficial gas velocity was 5 to $6 \mathrm{~cm} / \mathrm{sec}$.

It may be considered that a slight difference of the interfacial condition greatly affects the coalescence of bubbles and this results in the difference of the gas holdup. The discrepancy of the gas holdup between other researches and the present study will be attributed to the same reason.

\section{(2) Axial dispersion coefficient}

In Fig. 10 the axial dispersion coefficient is plotted as a function of the superficial gas velocity with the results of previous investigations ${ }^{2,7,8,9}$, which were determined from the liquid tracer method. Every result shows that the axial dispersion coefficient increases linearly with superficial gas velocity up to a certain value, and then changes its rate of increase near the point. For the present study this phenomenon arises at about $2 \mathrm{~cm} / \mathrm{sec}$, which corresponds to the transient point for the gas holdup.

When there are large bubbles, the liquid is lifted up by the bubbles and then flows, down around them. These repetitions result in vigorous mixing of the liquid phase. On the contrary, it may be possible to guess that when the bubbles are small the liquid flows down among the bubbles, and the liquid mixing is naturally slack unless the circulation of the liquid is provoked by the group of bubbles. These distinctions of the mixing mechanism of the liquid would be the reason for the difference in rate of increase of axial dispersion coefficient with superficial gas velocity.

The discrepancy of the initiation of aggregation or coalescence would be ascribed to the addition of electrolyte and the contamination of water.

\section{Conclusion}

According to the motion of a solid particle, it was found that there was no remarkable deviation of liquid flow in the bubble column.

The distribution function of axial displacements of a solid particle was described by the normal distribution function. From this fact the mixing mechanism of the liquid element was considered to be analogous to that of diffusion. The dispersion coefficient was determined.

From the photos, it was recognized that the superficial gas velocity had the same effect upon the gas holdup and the dispersion coefficient. The dynamic behaviour of bubbles, especially the coalescence of bubbles, had the controlling effect on the performance of the bubble column.

\section{Nomenclature}

$E \quad=$ axial dispersion coefficient

$F=$ probability distribution of displacements of a solid particle whithin a fixed short time interval $[1 / \mathrm{cm}]$

$r=$ radial distance

$t \quad=$ time

$\tau \quad=$ time interval

$U_{G}=$ superficial gas velocity

$z \quad=$ distance

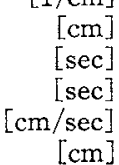


$z^{\prime} \quad=$ distance from the gas distributor

$\delta(\tau)=$ Dirac's delta function

$\sigma^{2}=$ variance

$\phi \quad=$ gas holdup

\section{Literature Cited}

1) Aoyama, Y., K. Ogushi, K. Koide and H. Kubota: J. Chem. Eng. Japan, 1, No. 2, 158 (1968)

2) Argo, W. B. and D. R. Cova: Ind. Eng. Chem. Process Design and Develop., 4, No. 4, 352 (1965) [cm] 3) Bischoff, K. B. and J. B. Phillips: ibid., 4, No. 4, 416 (1966)

4) Braulick, W. J., J. R. Fair and B. J. Lerner: A. I. Ch. E. Journal, 11, No. 1, 73 (1965)

5) Fair, J. R., J. Lambright and J. W. Andersen: Ind. Eng. Chem., Process Design and Develop., 1. No. 1, 33 (1962)

6) Koide, K., S. Kato, Y. Tanaka and H. Kubota: J. Chem. Eng. Japan, 1, No. 1, 51 (1968)

7) Reith, T., S. Renken and B. A. Israël: Chem. Eng. Sci, 23, 619 (1968)

8) Simes, W. and W. Weiss: Chem-Ing-Tech., 29, 727 (1957)

9) Tadaki, T. and S. Maeda: Kagak Kōgaku (Chem. Eng., Japan), 28, 270 (1964)

\title{
THE INTENSITY OF BULK FLOW IN A BUBBLE BED
}

\author{
HIROSHI YOSHITOME** AND TAKASHI SHIRAI \\ Research Laboratory of Resources Utilization, \\ Tokyo Institute of Technology
}

\begin{abstract}
The drag force acting against a spherical float was investigated to define the complex flow of gas-liquid mixture in a bubble bed, and was converted to the average intensity of bulk flow, $v_{z}$. The values of $v_{z}$ were found to be quite uniform over the central half cross-section of the bed and its directions were upward.

Over the outer annular cross-section, the values of $v_{z}$ were equal to those in the central section although their directions were downward.

Effects of various factors were further investigated, and a method for the calculation of $\mathbf{v}_{z}$ was also established.
\end{abstract}

\section{Introduction}

The bubble bed has been widely used in various fields of chemical industry for gas-liquid contacting operations. Various data in the literature for the bubble bed were, however, represented merely as empirical functions of the velocities of gas and of liquid only, and effects of factors such as liquid properties, type of apparatus and kind of gas-distributor have been left still uncertain.

The apparent intensity of bulk flow can be considered as one of the most basic data to be related to various operational factors such as mixing and diffusional rates in the bubble bed, including the above-mentioned effects.

This investigation was carried out to define and obtain the intensity of bulk flow, $v_{z}$, which has never been studied before, by measuring the drag force of a spherical float installed within a bubble bed without any continuous feed of liquid. A method for estimating $y_{z}$ at the center of the bubble bed from the data of critical gas velocity could also be established.

\section{Force Balance of a Spherical Float in the Bubble Bed, and the Determination of $\mathbf{v}_{\boldsymbol{z}}$}

The vertical force balance among the buoyancy force,

* Received on May 16, 1969 Presented at the 33rd Annual Meeting of the Society of Chemical Engineeres, Japan, April 1968

** Dept. of Bio-Chemical Engineering, Tokyo University of Education drag force and gravitational force to keep a spherical float at a middle point in a bubble bed can be represented by Eq. (1).

$$
(\pi / 6) D_{p}^{3} \rho_{L}(1-\varepsilon) g / g_{c}+F=W_{p}+W
$$

where $W$ is the balance weight to keep the spherical float halfway in the bubble bed.

The vertical force balance in a static liquid bed without aeration can be represented in the same way by Eq. (2).

$$
(\pi / 6) D_{p}{ }^{3} \rho_{L} g / g_{c}=W_{p}+W_{o}
$$

where $W_{o}$ is the balance weight for a spherical float in the static liquid bed.

From the above two equations, the drag force of a spherical float in the bubble bed, $F$, is represented by Eq. (3).

$$
F=(\pi / 6) D_{p}^{3} \rho_{L} \varepsilon g / g_{\mathrm{e}}+W-W_{\mathrm{o}}
$$

The intensity of bulk flow in the bubble bed, $v_{z}$, which corresponds to the drag force of the spherical float is defined by Eq. (4).

$$
F=C_{D} \frac{v_{z}^{2}\left[\rho_{L}(1-\varepsilon)+\rho_{G} \varepsilon\right]}{2 g_{c}}\left(\frac{\pi}{4}\right) D_{p}{ }^{2}
$$

where, $\rho_{L}(1-\varepsilon) \gg \rho_{G} \varepsilon$ in most cases.

The value of $v_{z}$ was calculated by Eq. (4), assuming that the drag coefficient of a sphere in the bubble bed was equal to that in the fluid flow of homogeneous phase at the same Reynolds number, $R e_{p}=D_{p} v_{a} \rho_{L}(1-\varepsilon) /$ $\mu_{L}$. The value of $v_{z}$ thus calculated includes many complex effects resulting from liquid properties, size and ascending velocity of bubbles, and the flow rate of gas. 